ARTICLE

\title{
On-chip spectrometers using stratified waveguide filters
}

Ang Li (iD ${ }^{1 凶} \&$ Yeshaiahu Fainman ${ }^{1}$

We present an ultra-compact single-shot spectrometer on silicon platform for sparse spectrum reconstruction. It consists of 32 stratified waveguide filters (SWFs) with diverse transmission spectra for sampling the unknown spectrum of the input signal and a specially designed ultra-compact structure for splitting the incident signal into those 32 filters with low power imbalance. Each SWF has a footprint less than $1 \mu \mathrm{m} \times 30 \mu \mathrm{m}$, while the $1 \times 32$ splitter and 32 filters in total occupy an area of about $35 \mu \mathrm{m} \times 260 \mu \mathrm{m}$, which to the best of our knowledge, is the smallest footprint spectrometer realized on silicon photonic platform. Experimental characteristics of the fabricated spectrometer demonstrate a broad operating bandwidth of $180 \mathrm{~nm}$ centered at $1550 \mathrm{~nm}$ and narrowband peaks with $0.45 \mathrm{~nm}$ Full-WidthHalf-Maximum (FWHM) can be clearly resolved. This concept can also be implemented using other material platforms for operation in optical spectral bands of interest for various applications.

\footnotetext{
${ }^{1}$ Department of Electrical and Computer Engineering, University of California at San Diego, La Jolla, CA, USA. ${ }^{凶}$ email: ang.li@nuaa.edu.cn
} 
O ptical spectrometers are indispensable elements in material science, chemical sensing, astronomical science, and in situ medical applications. The state-of-the-art high-performance spectrometers are currently realized by bulky, high-cost systems, thereby, limiting their deployment in various system applications. For example, miniaturized spectrometers could be integrated into intelligent portable devices, such as smartphones and unmanned aerial vehicles, in support of overarching internet of things applications. Among various approaches to miniaturized spectrometers, silicon photonics is most promising due to its compatibility with low-cost CMOS manufacturing technology ${ }^{1}$. In addition, the silicon photonics approach provides ultra-high index contrast that allows an extremely compact device footprint in support of high integration density ${ }^{1}$ and broad transparent spectral bandwidth window between 1.1 $\mu \mathrm{m}$ and $2.2 \mu \mathrm{m}$. This approach can be further extended by employing other CMOS compatible materials such as $\mathrm{SiN}^{2-4}$ with a low propagation loss of about $1 \mathrm{~dB} / \mathrm{cm}$, enabling the efficient implementation of long delay lines which are essential for achieving high spectral resolution in various types of integrated spectrometers $^{5,6}$. Moreover, with a hybrid integration approach, it is possible to integrate high-performance light source ${ }^{7-9}$, highspeed photodetectors ${ }^{10,11}$, electronic driver, and electronic signal processing circuits in a single chip-based package, leading to integrated miniaturized spectrometer systems for various practical applications ${ }^{12,13}$.

Numerous approaches have been carried out to realize highperformance spectrometers integrated on a silicon platform. The most straightforward approach is to disperse the spectral content of the incident signal into an array of photodetectors and record the corresponding spectral signals ${ }^{14-20}$. The dispersion can be achieved by an array of narrowband filters or dispersive elements such as gratings as shown in Fig. 1a, b. Although these types of

(a)

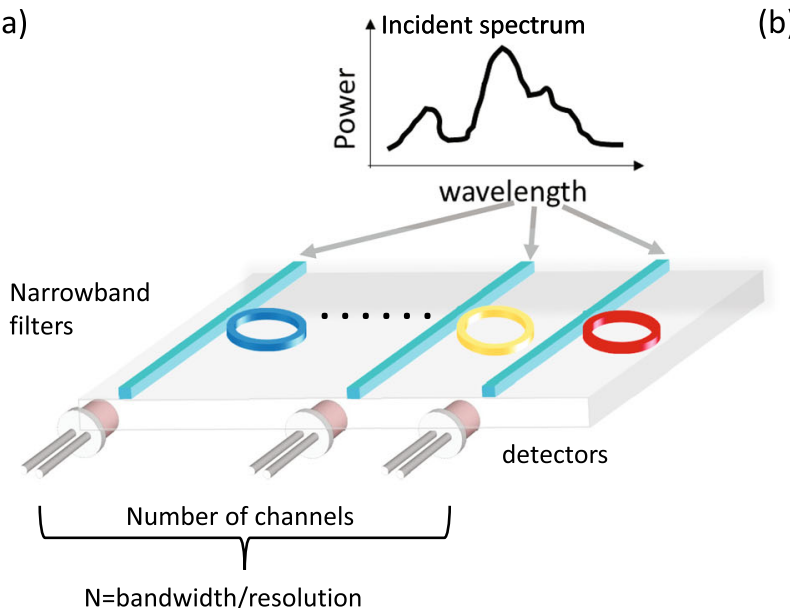

(b)

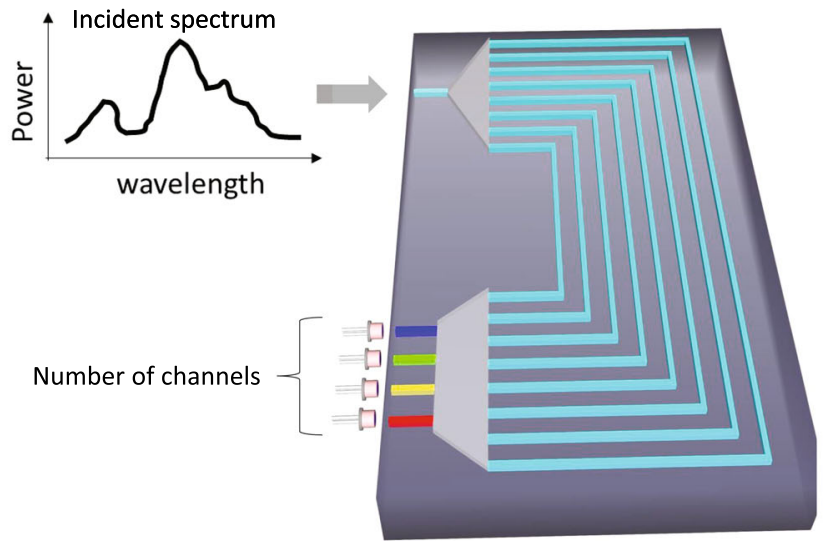
bandwidth signals and provide high spectral resolution, it will be achieved at the expense of introducing a very large number of building blocks (such as grating channels or filters and highperformance photodetectors), which in turn will result in considerably large footprint, high insertion loss, hardware cost, and operation complexity. For instance, echelle grating-based spectrometer with a footprint as large as $3 \times 3 \mathrm{~mm}^{2}$ can produce a resolution of $\delta \lambda=0.5 \mathrm{~nm}$, but it requires a large number of channels $N$ (e.g., over 120) to achieve moderate wavelength bandwidth of $\Delta \lambda=60 \mathrm{~nm}$ since the frequency bandwidth, $\Delta \omega=$ $N \delta \omega$ which reduces to $\Delta \lambda=N \delta \lambda^{18}$. Similar issues apply to ring resonators array-based spectrometer, which employs as many as 84 ring resonators to produce a resolution of $0.6 \mathrm{~nm}$ supporting the total bandwidth of $50 \mathrm{~nm}^{19}$. Both performance indicators are non-trivial to improve since the bandwidth is fundamentally limited by the free spectral range (FSR) of silicon ring resonators whereas the resolution is directly proportional to the quality factor, $Q$ (3 dB bandwidth) of the individual ring resonator. Moreover, for ring resonators with a high $Q$ factor, the backscattering induced resonance-splitting will emerge and significantly deteriorate the spectrometer performance ${ }^{21}$. However, the most critical concern of this class of spectrometers is their poor signal-to-noise ratio (SNR). Since the incident signal is divided into multiple narrowband signals to achieve the required spectral resolution, the power in each detection channel, linearly proportional to the ratio of bandwidth over the resolution, will be affected by the finite dynamic range and SNR of each corresponding photodetectors leading to poor SNR in the reconstructed broadband spectrum of the input signal, especially for the spectral components with very low magnitude. Another drawback lies in the poor tolerance to inevitable fabrication imperfections, which would introduce phase errors to multi-
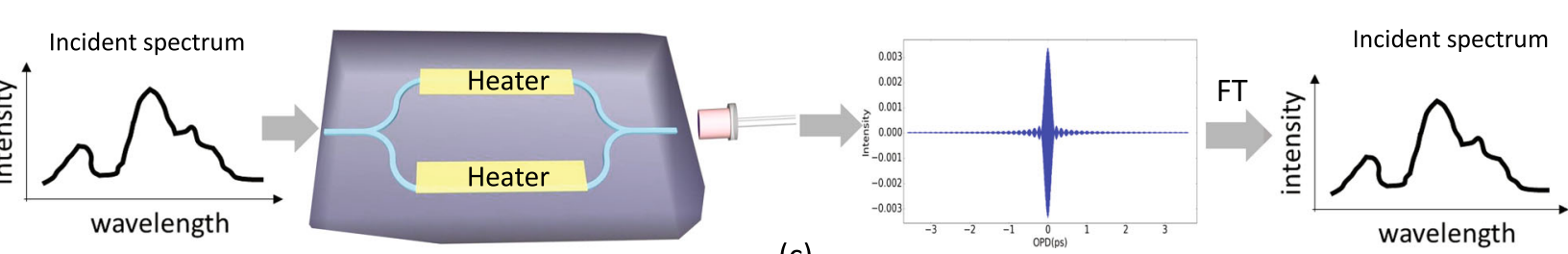

Fig. 1 Conceptual illustrations of different types of spectrometers. A schematic diagram describing the conventional split-and-detect spectrometers consisting of (a) narrowband filters array or (b) dispersive grating for fast spectrum reconstruction. The number of required channels is linearly proportional to the ratio of reconstruction bandwidth and resolution. The Fourier transform spectrometer (FTS) implemented on silicon photonics platform shown conceptually in (c) is used to generate and detect an interferogram (i.e., the autocorrelation of the input signal displayed by the left part of (c)) by changing optical path delay (OPD) between two arms of a balanced Mach-Zehnder-Interferometer (MZI) using integrated heaters. The spectrum of the input signal on the right part of (c) can be retrieved by performing a Fourier transformation (FT) of the interferogram. 
(a)

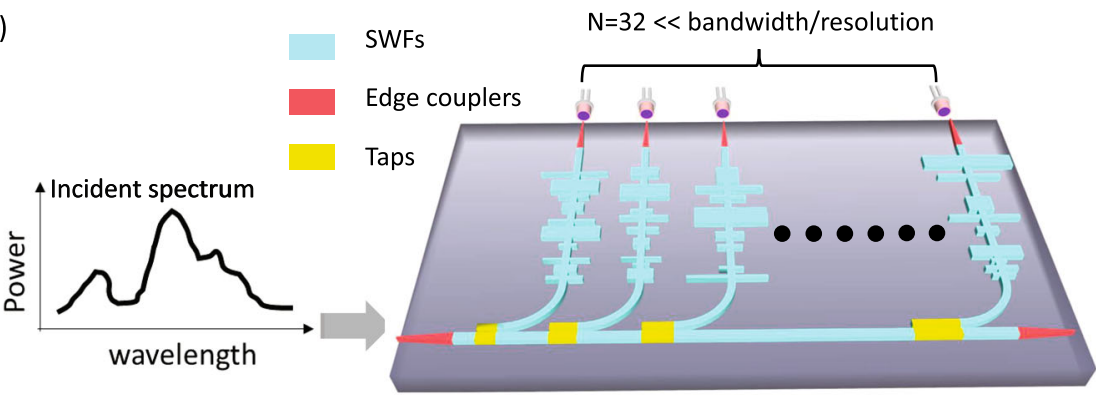

(b)

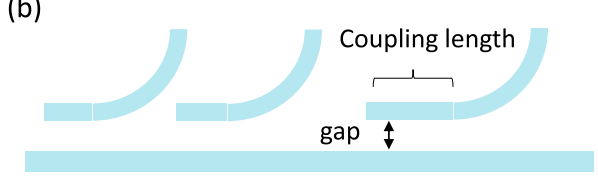

(c)
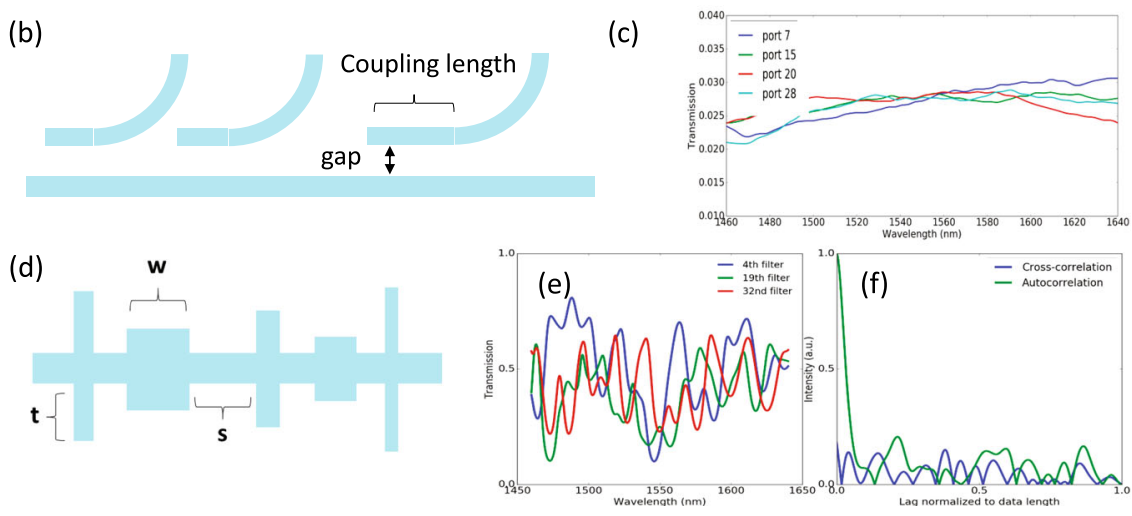

Fig. 2 Illustration of single-shot spectrometer enabled by broadband stratified waveguides filters (SWFs). a Depicts the simplified schematic of our implementation of this system on silicon. It contains two core parts: a $1 \times 32$ splitter based on cascaded taps and 32 broadband stratified waveguides filters (SWFs) with ultra-compact footprint and diverse spectral features. The number of channels needed is much smaller than in the split-and-detect concepts in Fig. 1a, b; (b) illustrates the structure for the cascaded taps with (c) showing the simulated transmission spectra at some output ports of the taps; (d) description of the SWF design with the defined design degrees of freedom $s, w$, and $t$ to control the transmission properties of the stratified waveguide; (e) presents the simulated transmission spectra of some of the designed SWFs and (f) shows the auto-correlation of the spectrum of a single SWF as well as the cross-correlation of two distinct SWFs.

channel gratings (such as arrayed waveguide grating ${ }^{14}$ ) and unpredictable resonance shifts to ring resonators, both of which are detrimental to the spectrometers' performance.

In contrast, due to Fellgett's advantage in terms of high $\mathrm{SNR}^{22}$, Fourier transform spectrometers (FTS) have been extensively investigated $^{23,24}$. The FTS principle of operation is based on detection of the auto-correlation function, or in other words, temporal interferogram of the incident signal by continuously tuning the optical path delay (OPD) between two pathways of the incident signal in an interferometer, and measuring the interference intensity at each OPD as illustrated in Fig. 1c. This type of spectrometer has been realized on a silicon photonic platform using a Mach-Zehnder-Interferometer (MZI) structure with integrated heaters used to change the waveguide effective index and, consequently, the OPD between the two arms of the MZI. This approach demonstrated operation with broadband input spectral signals (i.e., $\sim 60 \mathrm{~nm}$ ) and high resolution (i.e., sub nm) $)^{24}$, however, it was achieved at the expense of high power consumption of the heaters $(>5 \mathrm{~W})$, large driving voltage $(\sim 160 \mathrm{~V})$ and long measurement time $(>1.5 \mathrm{~h})$ due to slow thermo-optic effect. Moreover, the footprint of this realization is still considerably large (i.e., $>1 \mathrm{~mm}^{2}$ ).

Alternatively, a set of broadband filters with diverse spectral responses can be employed to sample the incident signal as schematically illustrated in Fig. $2 \mathrm{a}^{25,26}$. With a much smaller number of channels compared with the split-and-detect spectrometers, the incident spectrum can be reconstructed instantaneously using a computationally efficient signal processing algorithm. Intuitively, this approach is expected to have a much smaller than the FTS footprint and simultaneously provide a higher SNR in comparison to the split-and-detect spectrometers approaches. In addition, the set of broadband filter approach not only reduces the footprint compared to FTS but also does not require electrical driving/heating and long sampling time. The key challenge for the set of broadband filters approach is to develop a series of broadband filters with very different, ideally orthogonal, spectral response. Recent demonstrations of this type of singleshot spectrometers utilize either 195 colloidal quantum dots (CQD) absorption filters ${ }^{25}$ or 36 photonics crystals (PhC) cavities on Silicon-on-sapphire substrate ${ }^{26}$. Both approaches have good performance (a few hundred $\mathrm{nm}$ of bandwidth with 1-2 nm resolution), however, they do have individual challenges that are non-trivial to overcome. For the CQD-based approach ${ }^{25}$, the biggest challenge lies in the difficulty to integrate CQD into a CMOS compatible material platform supporting massive fabrication capability. Moreover, due to the similar filter response, a large number of filters (195) is needed to reconstruct a $300 \mathrm{~nm}$ bandwidth with a $2 \mathrm{~nm}$ resolution. In addition, the incident signal has to be split into 195 channels, which is even worse in terms of SNR compared to the split-detect approaches to achieve singleshot spectrum reconstruction. For the $\mathrm{PhC}$ cavities approach ${ }^{26}$, the incident light is coupled from free space modes into slab cavity modes experiencing an appreciable mode mismatch between a $210 \times 210 \mu \mathrm{m}^{2}$ aperture with corresponding free-space modes and highly confined $\mathrm{PhC}$ modes, resulting in a considerable coupling loss. Moreover, the performance of the spectrometer is very sensitive to the variation of the free space modes incidence angle, which, in practice, is difficult to accurately control. In addition, the reported configuration is integrated with a commercially available CMOS sensor, resulting in a large device volume and slow response.

In this manuscript, we propose and demonstrate experimentally a chip-scale single-shot spectrometer using stratified waveguides filters (SWF) on a silicon platform aiming at sparse 
spectrum reconstruction. Due to the rich design degrees of freedom, the SWFs can be constructed to produce spectral responses with very low cross-correlation, leading to high sampling accuracy of the incident signal. Consequently, only 32 filters are adequate for the detection and reconstruction of the sparse spectrum with good quality. In our experimental realization, each SWF has a footprint less than $1 \times 30 \mu \mathrm{m}^{2}$, much smaller compared with the work in ${ }^{26}$. In addition, we also construct an ultracompact uniform splitter to introduce the incident signal into the 32 SWFs. The core part of the $1 \times 32$ splitters and the 32 filters occupy a total area of about $35 \times 260 \mu \mathrm{m}^{2}$, which to the best of our knowledge, is the smallest footprint spectrometer implemented in a silicon photonic platform. The fabricated SWF-based spectrometer is interfaced with optical fiber with low coupling loss and characterized using a broadband, a narrow band, and a combination of a broad- and narrow-band input signal. It successfully reconstructs a broadband signal with $>60 \mathrm{~nm}$ spectral component and clearly resolves narrow peaks with full-widthhalf-maximum (FWHM) of $0.45 \mathrm{~nm}$.

\section{Results}

Principle and design. The detected power at a photodetector of an unknown signal with spectrum power $P(\lambda)$ passing through a broadband filter with transmittance $F(\lambda)$, can be mathematically written as:

$$
D=\int P(\lambda) F(\lambda) d \lambda
$$

where $D$ represents the detected power of the transmitted input signal. The transmission spectrum of the filter $F(\lambda)$ can be accurately measured during a calibration process. Ideally, we are dealing with continuous variables, however, during the reconstruction processes, we will reconstruct the digitized values of the input signal power spectrum. Therefore, for simplicity, we represent digitized values of both $P(\lambda)$ and $F(\lambda)$ by $1-\mathrm{D}$ signals, i.e., vectors with values $P\left(\lambda_{m}\right)$ and $F\left(\lambda_{m}\right)$, with $m=1,2 \ldots M$. The length of these vectors described by number $M$ determines the spectral resolution of the reconstructed input signal. With this transformation, Eq. (1) can be rewritten for the $n$th filter as:

$$
D_{n}=\sum_{m=1}^{M} P\left(\lambda_{m}\right) F_{n}\left(\lambda_{m}\right)
$$

Clearly, with $N$ distinct filters, we will generate $N$ corresponding values of $D_{n}$ with $n=1,2, \ldots N$. This formulation provides $N$ linear algebraic equations that can be solved to determine $M$ unknown values of the input signal, $P\left(\lambda_{m}\right)$. In a linear algebra formulation, the responses of $N$ filters yield,

$$
D_{N \times 1}=S_{N \times M} P_{M \times 1},
$$

where a sampling matrix $S_{N \times M}$ of size $[N \times M]$ connects the detected intensities vector $D_{N \times 1}$ of length $N$ with the input signal vector $I_{M \times 1}$ of length $M$. With a proper design, the number of filters required for reconstruction of the input signal can be much smaller than the ratio of the targeted bandwidth over the desired spectral resolution, i.e., $N \ll M$. Therefore, it outperforms previous split-and-detect spectrometers in multiple aspects including dynamic range, SNR, footprint, hardware cost, and system operation complexity. In comparison with FTS techniques, this approach has the advantages of no power consumption and the ability to instantaneously reconstruct the spectrum at the expense of reduced SNR. Note that, the rank of matrix $S$ needs to be as large as possible, in other words, the transmission spectra of $N$ filters have to be ideally orthogonal with zero cross-correlation. For the case of $N \ll M$, the Eq. (2) is a well-known underdetermined linear algebra problem, which can be solved using linear regression algorithm by minimizing $l_{2}$ norm of the Eq. $(3)^{27}$ :

$$
\text { minimize }\|D-S P\|^{2} \text { subject to } 0 \leq P \leq 1
$$

$D, S$, and $P$ are matrix representations described in Eq. (3). The importance to choose correct algorithms can be found in Supplementary note 1 of the supplementary document. For the case with stronger measurement noise, regularization of the $l_{2}$ norm of $P$ or standard deviation of $P$ can be added to the regression with a certain weight coefficient $\alpha$ for smooth spectrum reconstruction:

$$
\text { minimize }\|D-S P\|^{2}+\alpha\|P\|^{2} \text { subject to } 0 \leq P \leq 1
$$

The conceptual schematic of our implementation of a singleshot spectrometer system enabled by broadband filters is shown schematically in Fig. 2a. In general, such a type of spectrometer consists of two stages: splitting the incident signal into $N$ channels and sampling it by a set of $N$ broadband filters. To split the incident signal into multiple channels, the most straightforward approach is to use a multistage $Y$-junction tree topology, however, this can result in a large footprint. Besides, the number of output ports is limited to $2^{y}$, where $y$ refers to the number of stages of $Y$ junctions for our case $y=\log _{2} N$, with a total of $N \log _{2} N$ junctions. To reduce the footprint and achieve flexibility in determining the number of channels, we develop a splitter consisting of a series of cascaded $N$ taps attached to a common bus waveguide as shown in Fig. $2 \mathrm{~b}$ resulting in the entire footprint of a 32-port splitter as small as $5 \times 256 \mu \mathrm{m}^{2}$. The design efforts were put to individual tap to ensure a small imbalance among the transmission at each channel according to the following equation:

$$
\kappa_{N}=\frac{\kappa_{0}}{1-(N-1) \kappa_{0}},
$$

where $\kappa_{N}$ is the power coupling coefficient at the $N$ th tap and $\kappa_{0}$ is the desired transmission coefficient at each port, which for uniform splitting should be $\frac{1}{N}$. The simulated transmission spectra of the coupling regions for a few of the channels are shown in Fig. 2c. Clearly, each port extracts a similar amount of power from the common bus waveguide with very little non-uniformity across the flat regions which can always be accounted for during the calibration process. In supplementary note 2 , we also provide an analysis of how the spectrometer's performance will vary with power imbalance between different channels.

The core part of the spectrometer is the set of "highperformance" broadband SWFs. The term "high-performance" here is referring to two simultaneously achieved properties: (i) each SWF should produce a transmission spectrum with diverse features, in other words, the correlation length in the wavelength span (or minimum optical distance between two distinguishable wavelength points) should be small in order to provide high spectral resolution when sampling the input signal spectrum and (ii) the transmission spectra from any two SWFs should be very different (i.e., independent or orthogonal), in order to obtain a sampling matrix $S_{N \mathrm{X} M}$ with large rank. It is non-trivial to meet these two requirements while maintaining an ultra-compact footprint, simultaneously. A completely random or disordered medium has been reported to provide diverse spectral features and has been used to demonstrate hyperspectral imaging and ultra-compact spectrometers ${ }^{28-30}$. However, for random filters the light will be scattered by numerous defects instead of creating a well-confined, propagating mode in the medium, thereby leading to very high insertion loss for the input signal and consequently result in very low power collected by the detectors. Inspired by this effect, we combine the advantages of disordered medium and high-confinement silicon strip waveguide and develop SWF structure as a broadband filter. Our approach uses controlled perturbations of the Si waveguide by adjusting control 
parameters $(s, w, t)$ defined in Fig. 2 d. Stratified waveguides or stratified mediums have been studied for optical thin film filters, ultrasonic wave propagation, metallic optical fibers, fiber grating couplers, waveguide radiation, etc. ${ }^{31-35}$. Analogously, the control parameters $(s, w, t)$ determine the effective index and the effective thickness for each layer in the SWF and can be used to design the desired transmission spectrum property of each of the 32 filters. Each layer can be designed to have different parameters in terms of width and length corresponding to their effective indexes and thicknesses. Moreover, the spacing between the consequent layers can also be varied. With our SWF approach, we can exploit a large number of these design degrees of freedom to construct efficient broadband spectral transmission filters having diverse spectral features in contrast to previously investigated techniques such as CQD absorption filters and photonics crystal cavities ${ }^{25,26}$. While compared with a completely disordered medium, the high confinement from silicon waveguide assures high power collected by the photodetectors.

The filters' transmission spectra play a vital role in the spectrometer's performance (more details can be found in Supplementary note 3 of the supplementary document). We use autocorrelation and cross-correlation functions to characterize the filter's transmission spectra. The width of the autocorrelation illustrates how "fast and random" are the features in the transmission spectrum of the SWF, providing a design metric for achieving the desired resolution. Intuitively, our SWFs transmission functions should have spectral features (i.e., variations) on the same scale as the desired resolution which will be apparent in the width of the main lobe of the corresponding autocorrelation function for each SWF, which therefore can serve as the resolution metric during the design process. The cross-correlation between two distinct SWFs indicates how "different" they are from each other. In ideal cases, they should be orthogonal and the cross-correlation should be zero at each point. These desired transmission characteristics of SWFs were achieved by varying the parameters space considering the feature size of current technology, i.e., the parameters $s$ and $w$ are varied in the range of 100-200 nm, while $h$ is varying between $100 \mathrm{~nm}$ and $300 \mathrm{~nm}$ (see definitions in Fig. 2d). The SWF design contains 75-125 layers imposed on a standard silicon strip waveguide $(220 \mathrm{~nm} \times 450 \mathrm{~nm})$. All the design parameters $s, w, t$ were varied with an increment step of 5 $\mathrm{nm}$, which is a reasonable assumption for the state-of-the-art CMOS technology. As a consequence, each SWF has an ultracompact footprint around $1 \mu \mathrm{m} \times 30 \mu \mathrm{m}$, more than 30 times smaller than these reported in the previous approaches ${ }^{26}$. Simulated transmission spectra of few example SWFs are shown in Fig. 2e, f for the autocorrelation and the cross-correlation, respectively. Clearly, the spectra of those filters contain diverse features with very little cross-correlation signal. One minor issue with the current configuration is the reflections caused by individual SWF. However, the reflected portion of the light will only propagate towards the original input port and will not be reflected back again to induce cross-talk among different channels as there doesn't exist any reflective element in the pathway towards the input port. In future work, we could implement an identical set of SWFs operating with couplers to the reflected optical signal to recycle these reflected portions of light to further increase the efficiency as well as SNR.

Experimental characterization. The designed spectrometers were fabricated by applied nanotools using their standard multiproject-wafer (MPW) tape out. The SEM image of the overview of the entire spectrometer (excluding fiber couplers) is given in Fig. 3a. And the zoom-out views of the SWF and tap coupler are given in Fig. 3b, c. Their process uses Electron-beam lithography on a $220 \mathrm{~nm}$ thick silicon-on-insulator (SOI) wafer with a $2-3 \mu \mathrm{m}$ BOX layer, however, in our design we intentionally used parameters to also make the fabrication compatible with standard optical lithography. A 2- $\mu$ m oxide protection layer was deposited on top of the silicon structures. Inverted tapers with the flat spectral response and less than $5 \mathrm{~dB}$ insertion loss were used for fiber-to-chip coupling. The setup we used for characterization of the chip is a standard optical setup with lensed fibers to couple light in and out of the chip as we are using edge couplers as onchip fiber couplers to enable broadband operation. The chip is located on a holder with a temperature controller and vacuum holder that mechanically stabilizes the chip. The photodetector used in the experiments is an Agilent 81635A power sensor with a sensitivity of $-80 \mathrm{dBm}$. As a first step, we measure the transmission spectrum of each filter for calibration, using Agilent 8164B laser with a narrow linewidth output that can be varied from $1460 \mathrm{~nm}$ to $1640 \mathrm{~nm}$ with a step of about 9 pm (i.e., $~ 20,000$ data points in total). The resultant responses for some of the fabricated filters normalized to transmission in a straight waveguide are shown in Fig. 4a. The dense ripples are due to facet reflections at the fiber couplers. They do not affect the performance significantly due to their relatively small amplitude (less than $0.8 \mathrm{~dB}$ ) compared with the spectra features of the filters' spectra. Also, these measured transmitted signals were used to estimate the auto-correlation for a single filter and crosscorrelation of two randomly selected filters (see Fig. 4b), which show the expected trends.

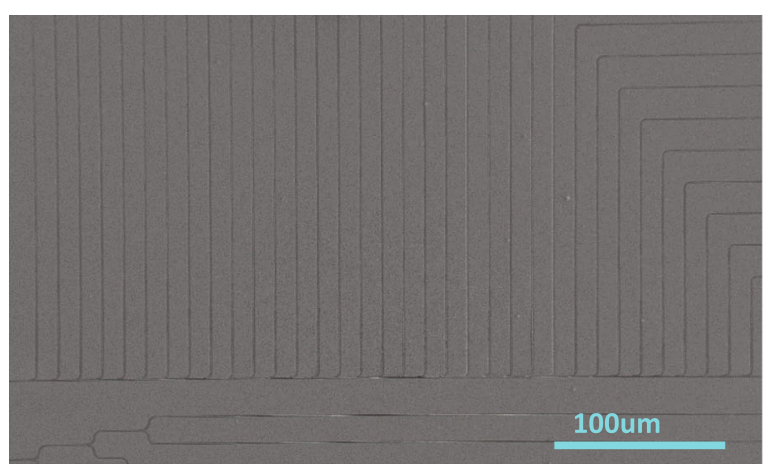

(a)

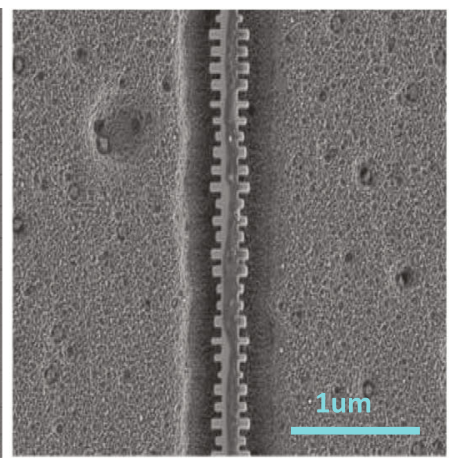

(b)

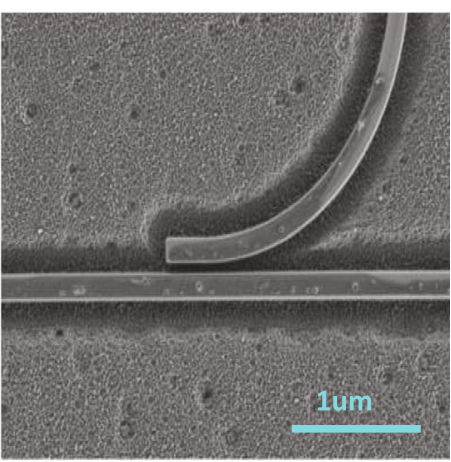

(c)

Fig. 3 Images of the fabricated devices. a Shows the SEM image of the overview of the entire spectrometer. $\mathbf{b}$, c Gives the zoom-out view of individual SWF as well as the tap-based splitter. 

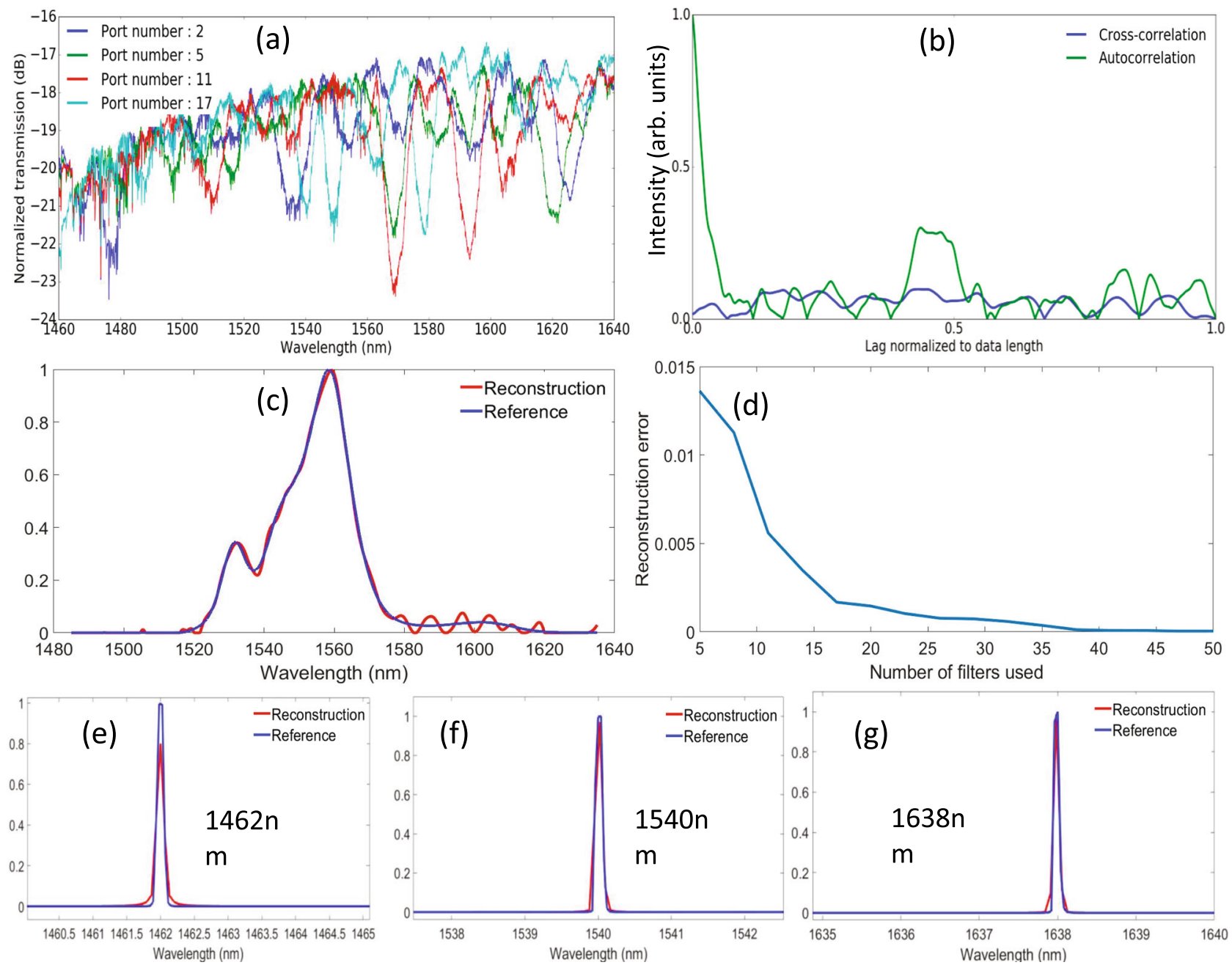

Fig. 4 Experimental validation and characterization of the single-shot SWF-based spectrometer system. a The measured spectra of some SWFs normalized to a straight waveguide transmission. Different colors indicate spectra of different SWFs. $\mathbf{b}$ The autocorrelation of a single filter measured spectrum (in green) and cross-correlation of two randomly chosen SWFs spectra (in blue). c Reconstruction of a broadband spectrum using the procedure described by Eqs. (4) and (5) (in red) and reference measured by a commercial optical spectrum analyzer (in blue). d Reconstruction errors as a function of the number of filters used for the input signal spectrum reconstruction. $\mathbf{e}-\mathbf{g}$ Reconstruction of the narrowband spectrum in the spectral range of $146 \mathrm{~nm}$ to $1640 \mathrm{~nm}$. The FWHM of the resolved peak maintains about $0.45 \mathrm{~nm}$ throughout the $180 \mathrm{~nm}$ span.

To test the performance of the fabricated SWF spectrometer, we first used a broadband spectrum from an ASE source as an input broadband signal with a bandwidth of over $60 \mathrm{~nm}$ centered around $1550 \mathrm{~nm}$. The output of the ASE source was introduced into our spectrometer and the intensity at the output of each filter was detected. To enable the signal processing of the detected signals, each individual SWF spectrum shown in Fig. 4a was digitized into a $1-\mathrm{D}$ array containing 450 uniform sampling points in the spectral window from $1485 \mathrm{~nm}$ to $1635 \mathrm{~nm}$, such that the incident signal spectrum will be represented by a 1-D array containing 450 unknown coefficients. This number of sampling points can be increased to achieve higher resolution at the cost of longer processing time (using CVX optimization algorithm implemented in MATLAB environment on a desktop with 4-core processors and 16 GB RAM consumes about $0.65 \mathrm{~s}$ to reconstruct the broadband ASE spectrum). Before performing the reconstruction of the broadband ASE spectrum, the regularization coefficient needs to be pre-calibrated. It is done by sending a broadband superluminescent laser diode (SLD) spectrum with over $80 \mathrm{~nm}$ spectral components around $1550 \mathrm{~nm}$ and adjusting the regularization coefficient until a good reconstruction appears (more details can be found in note 4 of the supplementary document). The reconstructed spectrum of the ASE source using the signal processing procedure described in Eqs. (2-5) with calibrated regularization coefficient is shown in Fig. $4 \mathrm{c}$ together with the reference curve obtained by using a commercial optical spectrum analyzer (OSA). The overall reconstruction is found in good agreement with the reference spectrum except for the lowamplitude tail in the optical range longer than $1580 \mathrm{~nm}$. This occurs due to the fact that the spectral components with higher amplitude have higher weights than the components with lower amplitude when solving Eq. (2). This is a common disadvantage for any multiplexing spectrometers. However, this deficiency can be effectively overcome by using a larger number of filters or a priori knowledge about the constraints on the incident signal or using segmented spectrometers, each of which only reconstructs a sub-band of the incident signal. In order to determine how many filters are necessary for broadband spectrum reconstruction, we also fabricated 50 stand-alone SWFs. The reconstruction errors as a function of the number of filters used for spectrum reconstruction are shown in Fig. 4d. Clearly, using more filters leads to a better quality of reconstruction, but at the expense of lower input power (i.e., a larger number of channels) and, consequently, lower SNR. 
Next, we test the capability to reconstruct narrowband components of the SWF spectrometer by sending narrow linewidth spectra from a tunable laser source. For the narrowband spectrum reconstruction, the corresponding regularization coefficient is calibrated by sending peaks at ten different locations (from $1460 \mathrm{~nm}$ to $1640 \mathrm{~nm}$ ) and adjust the corresponding coefficient for best reconstruction results. Then the average of the ten values is set as the common regularization coefficient for narrowband spectral components. In total, we performed over ten measurements with varying wavelengths. Three examples with spectral lines at $1462 \mathrm{~nm}, 1540 \mathrm{~nm}$, and $1638 \mathrm{~nm}$ are given in Fig. 4e-g. The achieved FWHM of the resolved peak at different wavelength locations maintains about $0.45 \mathrm{~nm}$ within the $180 \mathrm{~nm}$ optical range. The reconstruction results are shown in Fig. $4 \mathrm{e}-\mathrm{g}$ prove its ability to resolve narrow spectral components with resolution up to $0.45 \mathrm{~nm}$. And the operation bandwidth covers $180 \mathrm{~nm}$ which is limited by our measurement equipment. Ideally, the spectrum for calibration and reconstruction should be as distinct as possible, however, due to the limitations of our lab, we have to use the same source for both procedures.

Besides, we also investigate how the spectrometer can handle mixed spectrum, where both broadband spectrum and narrowband spectrum are present. To synthesize such an input signal, we use a $3 \mathrm{~dB}$ coupler to combine the output from the narrowlinewidth tunable laser source and the output from the $\mathrm{C}+\mathrm{L}$ band ASE (broadband spectrum) sources. This combined signal is then introduced to the input of the spectrometer. As mentioned above, the regularization weight $\alpha$ in Eq. (5) is different for the reconstruction of a broadband spectrum signal and narrowband spectrum signal. Therefore, if we use the term a optimized for broadband spectrum, the reconstruction quality of the narrowband signal peak is poor as shown in Fig. 4a. However, we can use segmented regularization in Eq. (5) with different weights a for broad and narrow spectral components:

minimize ||$D-S P\left\|^{2}+\alpha_{1}\right\| P_{1}\left\|^{2}+\alpha_{2}\right\| P_{2} \|^{2}$ subject to $0 \leq P_{1,2} \leq 1$,

where the to-be-reconstructed $1-\mathrm{D}$ array $\mathrm{P}$ is separated into two parts $P_{1}$ and $P$, which represent the narrow and broad spectral components respectively and $\alpha_{1}, \alpha_{2}$ refer to the optimized regularization weights for narrow and broad spectral components reconstruction, respectively. The two terms $\alpha_{1}$ and $\alpha_{2}$ can be accurately pre-determined using an extra calibration procedure by sending known narrowband and broadband signal into the spectrometer separately and adjust the corresponding weight term. Consequently, with this approach, the overall input spectrum signal can be reconstructed with good quality as demonstrated by the reconstructed results of spectra consisting of ASE spectrum and narrow spectrum peak at three different locations (see Fig. 4b-d).
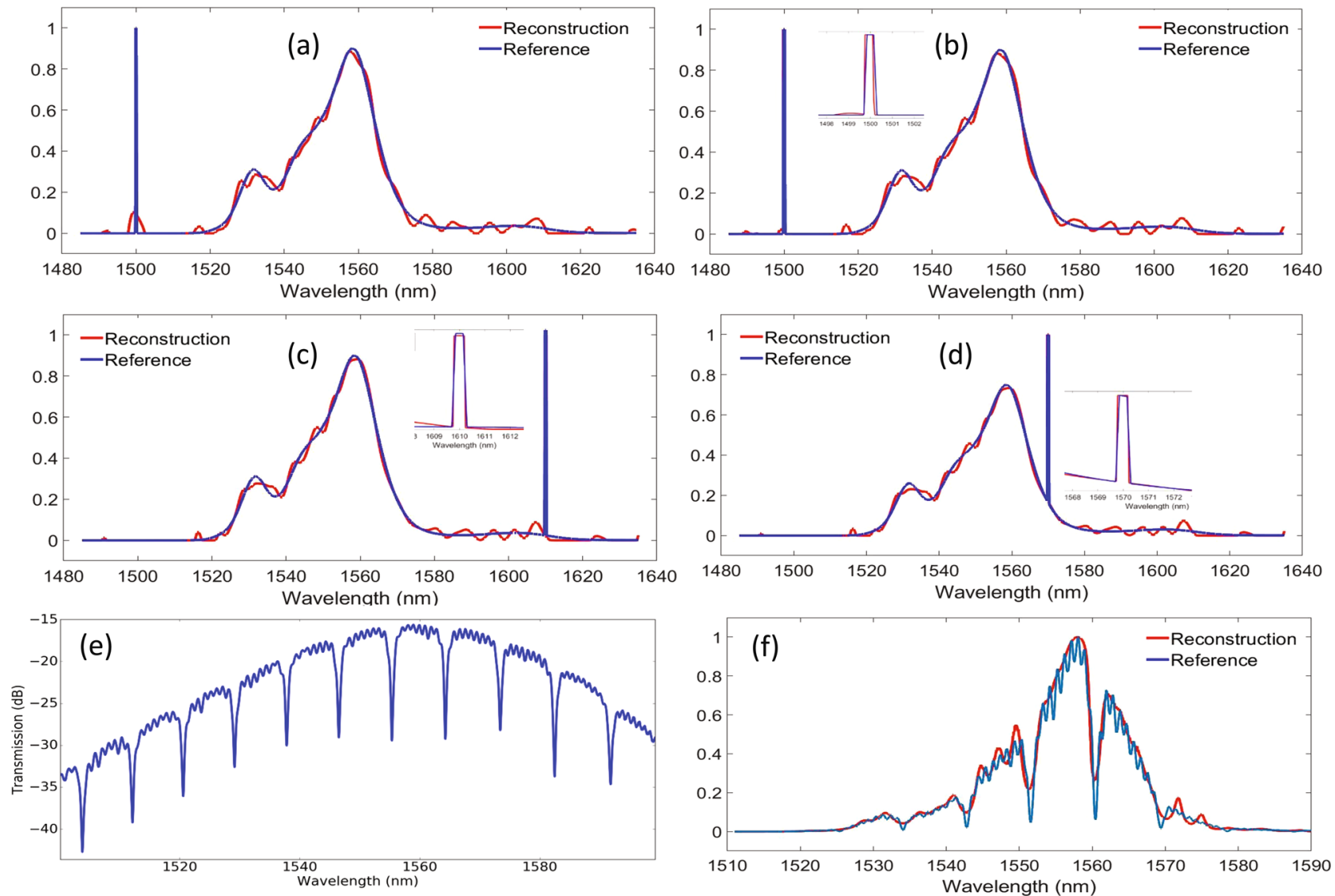

Fig. 5 Experimental validation and characterization of the single-shot SWF-based spectrometer for hybrid spectra that contain both broad spectral components (e.g., ASE source) and narrow spectral peak or notches. a Results obtained without further optimization of the reconstruction algorithm using Eqs. (4-5) showing poor quality in revealing the narrow spectral peak signals and the reference from a commercial optical spectrum analyzer (in blue). b-d Results obtained from using advanced reconstruction algorithm of Eq. (7) showing good reconstruction quality of all the three narrow spectral peak signals at different locations. Insets in (b-d) show the zoom-in area around the narrow line signals. e shows the notches generated by a ring resonator at a different chip. $\mathbf{f}$ Plots the reconstruction of the broadband spectrum with narrow notches (in cyan). Even if the performance compared degrades compared with a reconstruction of pure broadband spectrum, but the individual notches and the overall envelope can be accurately captured. 
This type of spectrometer is well known to be able to reconstruct sparse spectrum accurately, but we also investigate how the spectrometer can handle dense spectrum. We take the challenge of sending broadband spectrum with narrow notches to further test the capability of our spectrometer. This kind of signal is generated by first sending the ASE spectrum to a separate chip that has ring resonators to introduce notches. This chip is located in a separate testing setup that uses grating couplers and standard single-mode fibers as fiber/chip interface, then the output from the chip with the ring is sent to the spectrometer chip which is located in a different testing setup that uses edge couplers and lensed fibers to allow broadband testing. The measured transmission spectrum of the ring resonator using a tunable laser source is plotted in Fig. 5e, showing that the fabricated ring resonator has a measured FWHM of about $0.9 \mathrm{~nm}$ and an FSR of about $9 \mathrm{~nm}$. The ripples in Fig. 5e are due to parasitic reflections at the grating couplers as well as fiber facets. The measured transmission of the ASE source through the ring resonator chip using a commercial OSA is shown in Fig. $5 \mathrm{f}$ (blue curve) and the corresponding reconstruction obtained from the spectrometer chip is shown in Fig. $5 f$ (red curve). Clearly, the individual notch can be accurately reconstructed. Compared with reconstructions of narrow peaks and broadband spectrum, the mismatch, in this case, is clearer, as for this type of an underdetermined problem, solving a sparse matrix (i.e., corresponding to a spectrum only containing narrow peaks) is well-known to be easier than the dense matrix (i.e., corresponding to a broadband spectrum with narrow notches).

Note that, the measurement and characterization errors also impact the spectrometer performance. The potential contributions to the errors could be temperature variation between device calibration and spectrum reconstruction, as it will cause a shift of the filters' transmission spectrum and lead to inaccurate preparation of the sampling matrix ( $S$ in Eq. (3)) when performing spectrum reconstructions. We use a temperature controller to avoid this issue. Mechanical instability of the chip and the fibers could also contribute to measurement errors. We use a vacuum pump to stabilize the chip, and a fiber holder to stabilize the fibers. The setup is also pre-calibrated to ensure no fiber drift takes place within reasonable measurement time. In real applications, the spectrometer could be packaged with fiber arrays as optical $I / O$ to fully avoid these instabilities.

\section{Discussion}

In this manuscript, we implement a concept of a single-shot spectrometer that uses an array of broadband SWFs realized in silicon photonics platforms. The 32 SWFs are utilized as the broadband filters to sample the incident signal exploiting the multiple design degrees of freedom that enable the design and fabrication of spectral filters with diverse spectral features that possess narrow autocorrelation for high resolution and low crosscorrelation between the different filters in the array to increase orthogonality for effective signal reconstruction. In order to distribute the incident spectral signal into 32 filters with little power imbalance, we also developed and demonstrated experimentally an ultra-compact splitter based on cascaded taps. The total footprint of the splitter and the 32 SWFs is as small as $35 \mu \mathrm{m} \times$ $260 \mu \mathrm{m}$, which to the best of our knowledge, is the smallest experimentally demonstrated spectrometer on a silicon photonic platform. The experimental results demonstrate operation with broad bandwidth input signals (i.e., $180 \mathrm{~nm}$ centered at $1550 \mathrm{~nm}$ ), narrowband signals (i.e., $0.45 \mathrm{~nm}$ FWHM laser emission), and mixed broad/narrow-band input signals. In consistency with similar types of spectrometers, it shows better performance when reconstructing sparse spectrum while the performance degrades when dealing with dense spectra. While the concept is demonstrated in the optical range around $1550 \mathrm{~nm}$, its realization can be easily extended to other material platforms operating in the other desired optical spectral (e.g., SiN for operation in the visible and mid-infrared range). The SWF spectrometer approach is a promising candidate for cost-effective manufacturing of miniaturized spectrometers making them a suitable candidate for integration with various mobile and portable systems.

\section{Data availability}

The data that support the finding of this study are available from the corresponding author upon reasonable request.

Received: 17 February 2020; Accepted: 25 March 2021; Published online: 11 May 2021

\section{References}

1. Soref, R. The past, present, and future of silicon photonics. IEEE J. Sel. Top. Quant. Electron. 12, 1678-1687 (2006).

2. Baets, R. et al. (eds) Silicon photonics: silicon nitride versus silicon-on-insulator. Proc. Optical Fiber Communication Conference; 2016: Optical Society of America.

3. Khan, S., Chiles, J., Ma, J. \& Fathpour, S. Silicon-on-nitride waveguides for mid-and near-infrared integrated photonics. Appl. Phys. Lett. 102, 121104 (2013).

4. Rahim, A. et al. Expanding the silicon photonics portfolio with silicon nitride photonic integrated circuits. J. Lightw. Technol. 35, 639-649 (2017).

5. Bauters, J. F. et al. Silicon on ultra-low-loss waveguide photonic integration platform. Opt. Express 21, 544-555 (2013).

6. Dong, P. et al. Low loss shallow-ridge silicon waveguides. Opt. Express 18, 14474-14479 (2010).

7. Koch, B. R. et al. (eds). Integrated silicon photonic laser sources for telecom and datacom. Proc. National Fiber Optic Engineers Conference; 2013: Optical Society of America.

8. Chen, S. et al. Electrically pumped continuous-wave III-V quantum dot lasers on silicon. Nat. Photon. 10, 307 (2016).

9. Yang, H. et al. Transfer-printed stacked nanomembrane lasers on silicon. Nat Photon. 6, 615 (2012).

10. Asghari, M. \& Krishnamoorthy, A. V. Silicon photonics: energy-efficient communication. Nat. Photon. 5, 268 (2011).

11. Chen, L., Preston, K., Manipatruni, S. \& Lipson, M. Integrated GHz silicon photonic interconnect with micrometer-scale modulators and detectors. Opt Express 17, 15248-15256 (2009).

12. Lim, A. E.-J. et al. Review of silicon photonics foundry efforts. IEEE J. Sel. Top Quant. Electron. 20, 405-416 (2013)

13. Orcutt, J. S. et al. Open foundry platform for high-performance electronicphotonic integration. Opt. Express 20, 12222-12232 (2012).

14. Cheben, P. et al. A high-resolution silicon-on-insulator arrayed waveguide grating microspectrometer with sub-micrometer aperture waveguides. Opt. Express 15, 2299-2306 (2007).

15. Ryckeboer, E. et al. Silicon-on-insulator spectrometers with integrated GaInAsSb photodiodes for wide-band spectroscopy from 1510 to $2300 \mathrm{~nm}$. Opt. Express 21, 6101-6108 (2013).

16. Muneeb, M. et al. III-V-on-silicon integrated micro-spectrometer for the $3 \mu \mathrm{m}$ wavelength range. Opt. Express 24, 9465-9472 (2016).

17. Malik, A. et al. Germanium-on-silicon planar concave grating wavelength (de) multiplexers in the mid-infrared. Appl. Phys. Lett. 103, 161119 (2013).

18. Ma, K., Chen, K., Zhu, N., Liu, L. \& He, S. High-resolution compact on-chip spectrometer based on an echelle grating with densely packed waveguide array. IEEE Photonics. IEEE Photon. J. 11, 1-7 (2018).

19. Xia, Z. et al. High resolution on-chip spectroscopy based on miniaturized microdonut resonators. Opt. Express 19, 12356-12364 (2011).

20. Vasiliev, A. et al. Integrated silicon-on-insulator spectrometer with single pixel readout for mid-infrared spectroscopy. IEEE J. Sel. Top. Quant. Electron. 24, 1-7 (2018).

21. Li, A., Van Vaerenbergh, T., De Heyn, P., Bienstman, P. \& Bogaerts, W. Backscattering in silicon microring resonators: a quantitative analysis. Laser Photon. Rev. 10, 420-431 (2016).

22. Fellgett, $\mathrm{P}$. On the ultimate sensitivity and practical performance of radiation detectors. JOSA 39, 970-976 (1949).

23. Souza, M. C., Grieco, A., Frateschi, N. C. \& Fainman, Y. Fourier transform spectrometer on silicon with thermo-optic non-linearity and dispersion correction. Nat. Commun. 9, 665 (2018) 
24. Li, A., Davis, J., Grieco, A., Alshamrani, N. \& Fainman, Y. Fabrication-tolerant Fourier transform spectrometer on silicon with broad bandwidth and high resolution (2019)

25. Bao, J. \& Bawendi, M. G. A colloidal quantum dot spectrometer. Nature 523, 67 (2015).

26. Wang, Z. et al. Single-shot on-chip spectral sensors based on photonic crystal slabs. Nat. Commun. 10, 1020 (2019).

27. Grant, M., Boyd, S. \& Ye, Y. CVX: Matlab software for disciplined convex programming. 2008.

28. Redding, B., Liew, S. F., Sarma, R. \& Cao, H. Compact spectrometer based on a disordered photonic chip. Nat. Photonics 7, 746 (2013).

29. Hartmann W. et al. Waveguide-integrated broadband spectrometer based on tailored disorder. Adv. Opt. Mater. 1901602 (2020)

30. Boniface, A., Gusachenko, I., Dholakia, K. \& Gigan, S. Rapid broadband characterization of scattering medium using hyperspectral imaging. Optica 6, 274-279 (2019).

31. Galejs, J. Admittance of a waveguide radiating into stratified plasma. IEEE Trans. Antennas Propag. 13, 64-70 (1965).

32. Li, Y.-F. \& Lit, J. W. Guided even and odd modes in symmetric periodic stratified dielectric waveguides. JOSA A 5, 1050-1057 (1988).

33. Shihong, Z. Acoustic vector fields propagation in horizontal-stratified waveguide. J. Harbin Eng. Univ. 25, 38-42 (2004).

34. Tsao, C. Y., Payne, D. N. \& Li, L. Modal propagation characteristics of radially stratified and D-shaped metallic optical fibers. Appl. Opt. 28, 588-594 (1989).

35. Wang, B., Jiang, J., Chambers, D. M., Cai, J. \& Nordin, G. P. Stratified waveguide grating coupler for normal fiber incidence. Opt. Lett. 30, 845-847 (2005).

\section{Acknowledgements}

This work was supported by the Defense Advanced Research Projects Agency (DARPA) DSO NLM and NAC Programs, the Office of Naval Research (ONR), the National Science Foundation (NSF) grants DMR-1707641, CBET-1704085, NSF ECCS-180789, NSF ECCS-190184, NSF ECCS-2023730, the Army Research Office (ARO), the San Diego Nanotechnology Infrastructure (SDNI) supported by the NSF National Nanotechnology Coordinated Infrastructure (grant ECCS-2025752), the Quantum Materials for Energy Efficient Neuromorphic Computing-an Energy Frontier Research Center funded by the U.S. Department of Energy (DOE) Office of Science, Basic Energy Sciences under award \# DE-SC0019273. Advanced Research Projects Agency-Energy (LEED: A Lightwave Energy-Efficient Datacenter), and the Cymer Corporation.

\section{Author contributions}

A.L. conceived the idea, performed the simulation, device design, experimental characterization, and data analysis. Y.F. designed the experimental steps, performed data analysis, and supervised the project. All authors contributed to the writing of the manuscript.

\section{Competing interests}

The authors declare no competing interests.

\section{Additional information}

Supplementary information The online version contains supplementary material available at https://doi.org/10.1038/s41467-021-23001-6.

Correspondence and requests for materials should be addressed to A.L.

Peer review information Nature Communications thanks Daoxin Dai, Zongfu Yu, and the other, anonymous, reviewer for their contribution to the peer review of this work.

Reprints and permission information is available at http://www.nature.com/reprints

Publisher's note Springer Nature remains neutral with regard to jurisdictional claims in published maps and institutional affiliations.

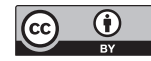

Open Access This article is licensed under a Creative Commons Attribution 4.0 International License, which permits use, sharing, adaptation, distribution and reproduction in any medium or format, as long as you give appropriate credit to the original author(s) and the source, provide a link to the Creative Commons license, and indicate if changes were made. The images or other third party material in this article are included in the article's Creative Commons license, unless indicated otherwise in a credit line to the material. If material is not included in the article's Creative Commons license and your intended use is not permitted by statutory regulation or exceeds the permitted use, you will need to obtain permission directly from the copyright holder. To view a copy of this license, visit http://creativecommons.org/ licenses/by/4.0/.

(C) The Author(s) 2021 